\title{
Modeling of hydrogen effects on short crack propagation in a metastable austenitic stainless steel (X2CrNi19-11)
}

\author{
Volker Schippl ${ }^{1, *}$, Sven Brück ${ }^{1}$, Hans-Jürgen Christ, Claus-Peter Fritzen ${ }^{2}$, Martina Schwarz ${ }^{3}$, and Stefan Weihe ${ }^{3}$ \\ ${ }^{1}$ Institut für Werkstofftechnik, Universität Siegen, 57068 Siegen, Germany \\ ${ }^{2}$ Institut für Mechanik und Regelungstechnik - Mechatronik, Universität Siegen, 57068 Siegen, Germany \\ ${ }^{3}$ Materialprüfungsanstalt, Universität Stuttgart, 70174 Stuttgart, Germany
}

\begin{abstract}
The experimentally observed short fatigue crack growth rate of an uncharged specimen tested in air is compared with results obtained from specimens tested in $10 \mathrm{MPa}$ hydrogen atmosphere and specimens previously charged with hydrogen. To further discuss the hydrogen related short propagation mechanisms, a simulation approach for predicting short fatigue crack growth is presented. The boundary element method is used for calculating stresses and displacements in an anisotropic elastic solid. The hydrogen concentration is assumed to be homogeneously distributed in the microstructure. Based on this modelling approach, it could be concluded that hydrogen leads to an increasing short fatigue crack growth rate due to increasing irreversible deformation processes at the crack tip and also promotes grain boundary cracking in specimens tested in $10 \mathrm{MPa}$ hydrogen atmosphere.
\end{abstract}

\section{Introduction}

Due to the global effort to reduce the emission of $\mathrm{CO}_{2}$, the need for renewable and sustainable energy resources arises. Hydrogen is a promising candidate in the automobile industry as a fuel with zero-emissions during the electrochemical reaction in the fuel cell.

During operation, the components responsible for transporting the hydrogen gas to the fuel cell experience cyclic loading due to service loading conditions. Thus, to safely design structural components with long product lives, the effect of hydrogen on the material behaviour during cyclic loading has to be considered. Commonly, materials are used which show little or no effect on hydrogen. This includes materials such as austenitic stainless steels containing a high amount of nickel, e. g. AISI 316. These austenitic stainless steels have a high resistance against corrosion and are very ductile, but are relatively expensive due to the high nickel content. In order to be able to safely use austenitic stainless steels with a lower nickel content, the effects of hydrogen on the fatigue damage has to be clarified. A lot of the published research highlights the influence of hydrogen on the fatigue damage in the long crack growth regime $[1,2]$. Fukuyama et al. investigated the fatigue properties of AISI 304 tested in hydrogen pressure up to 4.0 MPa [1]. They observed an increasing fatigue crack growth rate with increasing hydrogen pressure. Murakami et al. observed fatigue cracks up to $1000 \mu \mathrm{m}$ in specimens previously charged with hydrogen [2]. They have also shown that the presence of hydrogen leads to an increasing fatigue crack growth rate in these specimens.

Thus, it is well known that hydrogen can lead to a significant reduction of fatigue life by changing the crack propagation mechanisms in the long crack growth regime. But during cyclic loading in high cycle fatigue, the initiation and growth of microstructurally short cracks may be crucial to determine the fatigue life. It is well established that under these conditions, short crack growth can take up to $90 \%$ of the total fatigue life [3].

The crack propagation of short cracks is highly influenced by microstructural characteristics. For austenitic stainless steels under low amplitude loading, it could be observed that short cracks propagate along shear bands which formed at the crack tip due to locally high stresses. Wilkinson et al. proposed a theoretical model for describing the crack propagation of these microstructurally short cracks [4]. In 1996, they explained that the cyclic crack advance is caused by irreversible deformation processes at the crack tip. Dislocations and vacancy dipoles nucleating in the direction of the activated slip system in front of the crack tip are absorbed by the crack during cyclic loading leading to crack growth. During this crack propagation, grain and phase boundaries may act as barriers for dislocations and thus hinder the further propagation of slip bands/shear bands and short cracks to the next grain. This leads to a decreasing crack growth rate near grain boundaries and is characteristic for the stage I crack growth regime.

The question now arises how hydrogen changes the deformation and crack growth mechanisms. To elucidate

\footnotetext{
* Corresponding author: volker.schippl $@$ uni-siegen.de
} 
the mechanisms responsible for the observed increasing fatigue crack growth rate in the presence of hydrogen, a metastable austenitic stainless steel is tested as received in air as well as in $10 \mathrm{MPa}$ hydrogen atmosphere and in a condition precharged with hydrogen. With fatigue tests in these conditions, the short crack propagation mechanisms related to hydrogen can be further investigated and distinguished from those observed in laboratory air. The interpretation of these experimental observations will be supported by simulation studies on the short crack propagation. The simulation results will be validated by comparing the observed crack growth rates and crack paths in real microstructures. Furthermore, it is possible to relate these results to the proposed theories on the effects of hydrogen on the deformation and crack growth mechanisms.

A well accepted theory states a hydrogen-enhanced localized plasticity (HELP) mechanism [5]. Birnbaum et al. proposed that hydrogen "shields" the interaction between dislocations and short range stress fields and may also decrease the stability of dislocation dipoles. They further stated that this effect is pronounced where dislocations are closely packed e. g. at dislocation pileups. This is the case near grain boundaries or at the vicinity of a crack. Another established theory is the hydrogen-enhanced decohesion (HEDE) mechanism $[6,7,8]$. It is proposed that hydrogen e. g. residing at grain boundaries weakens the strength and facilitates cracking. It is further stated that this mechanism holds for hydrogen occupying interstitial normal lattice site where it decreases the interatomic potential. In 2004, Nagumo proposed the hydrogen-enhanced stress-induced vacancy (HESIV) mechanism [9]. The observed decreasing crack growth resistance in presence of hydrogen during static tensile tests in austenitic stainless steels AISI 304 and $316 \mathrm{~L}$ is explained by the increasing density of voids at the crack tip [10]. For a more detailed description on different theories on the effect of hydrogen on deformation processes, the reader is directed to an overview by Lynch [11].

The proposed theories described above can be seen as the fundamental mechanisms explaining the influence of hydrogen on deformation processes in the atomic scale which causes hydrogen related deformation mechanisms at the mesoscopic scale e. g. when short crack growth processes are observed. An example of this theoretical approach is the explanation of increased fatigue crack growth rate in the long crack growth regime in carbon steel JIS-SM490B by the hydrogenenhanced successive fatigue crack growth (HESFCG) model [12]. It is proposed that hydrogen accumulates at the crack tip causing a localization of slip deformation due to the HELP mechanism. This reduces the capability of the crack to blunt which further leads to successive crack growth.

The investigation of the short fatigue crack propagation in the presence of hydrogen builds upon previous work [13-17]. This study emphasises on relating the observed fatigue crack growth rates with respect to the testing configuration. With this approach, it is possible to reveal new insights into hydrogen-enhanced fatigue crack growth mechanisms at the mesoscopic scale and allows conclusions on the dominant fundamental hydrogen mechanisms acting in austenitic stainless steels.

In the following section a detailed description of the investigated material as well as on the experimental results is given. These observations lead to the presented short fatigue crack growth model used in this study which is solved by the boundary element method (BEM). The simulation results will be presented and discussed highlighting the acting hydrogen-enhanced short crack propagation mechanisms.

\section{Experimental results}

The investigated metastable austenitic stainless steel is based on the chemical composition of X2CrNi19-11 (AISI 304L) and is alloyed according to Table 1 where the yield strength $R_{\mathrm{p} 02}$, the calculated stacking fault energy (SFE) [18] as well as the $M_{\mathrm{d} 30}$-temperature [19] are given.

Three different fatigue tests were conducted: (i) on uncharged specimens in laboratory air, (ii) on precharged specimens in laboratory air and (iii) on uncharged specimens in $10 \mathrm{MPa}$ hydrogen atmosphere. The fatigue tests on uncharged and precharged specimens have been carried out using a miniature testing machine, further described in [20], at a test frequency of $f=1 \mathrm{~Hz}$, a stress ratio of $R=-1$ and with a stress amplitude $\sigma_{\mathrm{a}}=365 \mathrm{MPa}$. The geometry of the specimen used is shown in Fig. 1a. The fatigue tests in $10 \mathrm{MPa}$ hydrogen atmosphere were conducted on a specimen as shown in Fig. 1b applying a stress amplitude of $\sigma_{\mathrm{a}}=270 \mathrm{MPa}$, a test frequency of $f=1 \mathrm{~Hz}$ and a stress ratio of $R=-1$. a)

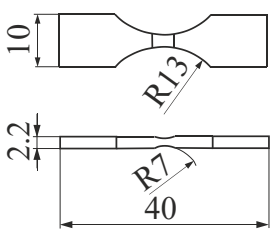

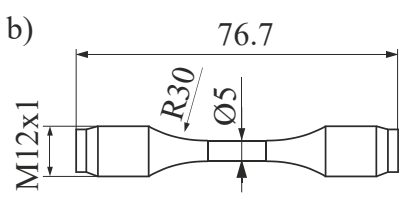

Fig. 1. (a) Geometry of the in situ specimen used for the uncharged and precharged fatigue tests and (b) specimen for fatigue tests in $10 \mathrm{MPa}$ hydrogen atmosphere

The precharged specimens were exposed to $10 \mathrm{MPa}$ hydrogen atmosphere at $180^{\circ} \mathrm{C}$ for 28 days. The hydrogen concentration was measured by the hot gas extraction method. A steady state hydrogen concentration of $c_{\mathrm{H}}=40 \mathrm{wppm}$ was reached resulting in a diffusion coefficient $D=1.8 \cdot 10^{-8} \mathrm{~cm}^{2} / \mathrm{s}$ which is in a good agreement with obtained results on AISI 304 [21].

Fig. 2 shows the experimentally observed crack path in

Table 1. Chemical composition (wt. \%), yield strength $\left(R_{\mathrm{p} 02}\right)$, stacking fault energy (SFE) and $M \mathrm{~d} 30$-temperatures of the material

\begin{tabular}{cccccccccccccc}
\hline $\mathrm{C}$ & $\mathrm{Si}$ & $\mathrm{Mn}$ & $\mathrm{P}$ & $\mathrm{S}$ & $\mathrm{Cr}$ & $\mathrm{Ni}$ & $\mathrm{Mo}$ & $\mathrm{Nb}$ & $\mathrm{Ti}$ & $\mathrm{N}$ & $R_{\mathrm{p} 02}[\mathrm{MPa}]$ & $\mathrm{SFE}\left[\mathrm{mJ} / \mathrm{m}^{2}\right]$ & $M_{\mathrm{d} 30}\left[{ }^{\circ} \mathrm{C}\right]$ \\
\hline 0.016 & 0.48 & 1.75 & 0.012 & 0.008 & 17.78 & 12.36 & 0.02 & 0.01 & 0.005 & 0.017 & 199 & 31.33 & -85.91 \\
\hline
\end{tabular}


the uncharged specimen (Fig. 2a-b), the precharged specimen (Fig. 2c-d) and the uncharged specimen in 10 MPa hydrogen atmosphere (Fig. 2e-f).

Cracks predominantly initated at grain boundaries in the uncharged specimen and in specimen in $10 \mathrm{MPa}$ hydrogen atmosphere as often observed in uncharged austenitic stainless steels [22, 23]. In precharged specimens, the crack initation site shifted towards triple junctions [24]. As earlier mention, hydrogen residing at grain boundaries could weaken the interfacial strength leading to premature fracture. This effect is more pronounced at triple junctions, thus leading to facilitated crack initiation at these sites.

The crack further propagates through grains in uncharged and precharged specimens in the absence of any or with partially very confined martensite formation at the crack tip. The crack paths in specimens in $10 \mathrm{MPa}$ hydrogen atmosphere cleary deviated from the crack paths observed in uncharged and in precharged specimens. The crack propgation is often restricted to grain boundaries. In uncharged and precharged specimens, the transgranular crack propagates by alternate sliding on two alternating slip systems as can be seen in Fig. 2a and c. These observations can be explained by the different distribution of hydrogen in the precharged specimens and in the specimens in $10 \mathrm{MPa}$ hydrogen atmosphere. In the precharged condition, a homogeneous steady-state hydrogen concentration at the surface was established due to the charging conditions. Thus, the difference of the hydrogen occupying normal interstitial lattice sites compared to the hydrogen residing at the grain boundaries is less significant. It follows that neither trans- nor intergranular crack growth is favored and the crack path is comparable to uncharged specimens such that the crack initation from triple junctions is followed by transgranular crack propagation.

For the specimens in $10 \mathrm{MPa}$ hydrogen atmosphere facilitated hydrogen entry at grain boundaries may play a key role in explaining the different crack propagation paths. This results in a locally increasing hydrogen content at grain boundaries during cyclic loading. Following the HEDE mechanism, this promotes the crack propagation along grain boundaries.

It can also be seen that during cyclic loading a localization of plastic strain occurs as indicated by the emerging slip markings on the specimen surface as often observed for steels with low stacking fault energies. To determine the influence of hydrogen on the slip morphology, the average slip band heigth and average distance between slip bands were determined in the
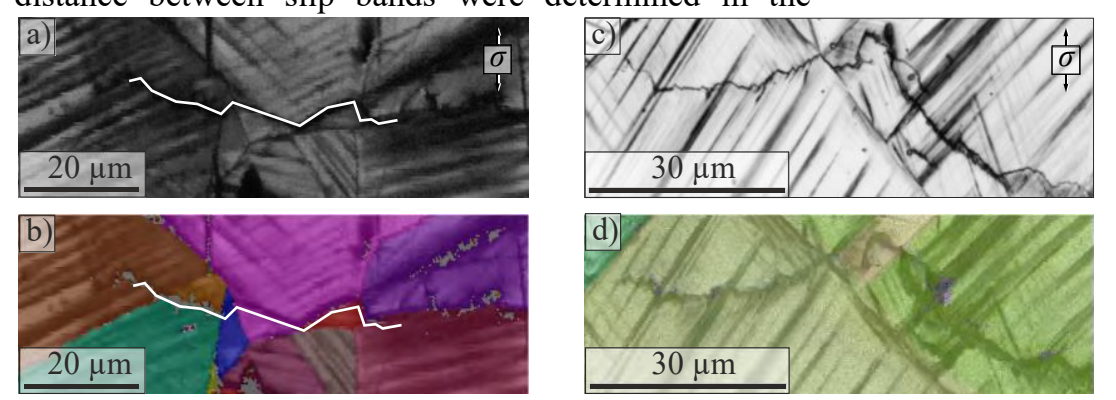

vicinity of the crack [24]. It could be observed that the maximum slip band heigth in precharged specimens $(0.14 \mu \mathrm{m})$ was significantly increased as compared to measurements in uncharged specimens $(0.08 \mu \mathrm{m})$. The average distance between slip bands increased from $1.92 \mu \mathrm{m}$ in uncharged specimens to $4.7 \mu \mathrm{m}$ in precharged specimens. Similar results could be obtained by Aubert et al. in AISI 316L [25] and by Abraham et al. in AISI 310s [26]. Both showed that in presence of hydrogen, an increase in slip band heigths and spacing of slip bands can be investigated. These observations further support the acting HELP mechanism in these metastable austenitic stainless steels. As hydrogen "shields" the interaction between dislocations and short range stress fields, the spacing between dislocation is reduced and the mobility of dislocations increases. This results in the observed increase in the maximum slip band heigth.

In the following section, the theory on modelling short fatigue crack propagation in the investigated austentic stainless steel is presented based on the mentionend experimental observations. The simulation emphasises on the deformation process in the vicinity of the crack. The influence of hydrogen on the crack propagation mechanisms will be revealed based on the simulation results of the predicted crack paths and crack growth rates of the experimentally observed short cracks depicted in Fig. 2.

\section{Simulation model}

In the simulation model, real microstructures are represented in the mesoscopic scale as a twodimensional anisotropic elastic solid including microstructural deformation processes. The investigated microstructure consists of several grains with distinctive crystallographic orientations and crystallographic structure of the corresponding phase which further determine the orientation of the different slip systems.

The propagation of microstructurally short cracks is significantly affected by microstructural characteristics such as grain or phase boundaries, the predominant tendency of dislocation structure arrangements such as evolving persistent slip bands and phase transformations processes e. g. the deformation induced martensite formation. In the simulation, the crack path is predicted starting from a pre-existing initial crack located at the nucleation site determined by scanning electron microscope (SEM) images. To predict the crack growth

Fig. 2. (a) Crack morphology and (b) EBSD scan of the uncharged specimen tested in laboratory air (crack depicted as a white line), (c), (d) of the precharged specimen $\left(c_{\mathrm{H}}=40 \mathrm{wppm}\right)$ and $(\mathrm{e}),(\mathrm{f})$ of the uncharged specimen in $10 \mathrm{MPa}$ hydrogen atmosphere 
in the simulation, two different crack propagation mechanisms have to be distinguished: (i) transgranular crack growth and (ii) intergranular crack growth.

The model for determining transgranular crack growth is based on the short crack growth model by Wilkinson et al. [4]. The crack growth rates of these microstructurally short cracks are calculated by relating the maximum and the minimum crack tip slide displacement during cyclic loading $\left(\triangle C T S D=C T S D^{\max }-C T S D^{\min }\right)$ to the crack growth rate $\mathrm{d} a / \mathrm{d} N[27]$

$$
\frac{\mathrm{d} a}{\mathrm{~d} N}=C_{\mathrm{CTSD}} \cdot \Delta C T S D
$$

where the parameter $C_{\mathrm{CTSD}}$ is a measure for the irreversible deformation processes at the crack tip. The crack tip slide displacement (CTSD) is mainly driven by the shear deformation along slip bands nucleating at the crack tip. Thus, only these shear bands are modelled in the simulation including only short range effects on the crack deformation processes. To model the shear band evolution during cyclic loading, the nucleation of these shear bands as well as the sliding along these shear bands is explicitly modelled. For nucleating shear bands at the crack tip, it is assumed that a critical shear stress $\left(\tau^{\mathrm{c}}\right)$ has to be exceeded. The plastic shear deformation along these shear bands is realised by applying an ideally elastic-plastic material law with a characteristic critical friction stress $\left(\tau^{\mathrm{F}}\right)$. It has further to be mentioned that only up to two slip systems are allowed to nucleate at the crack tip in a grain as commonly experimentally observed, e. g. see Fig. 2c. A slide deformation along these shear bands significantly alters the crack tip slide displacement $C T S D$ and the crack growth rate $\mathrm{d} a / \mathrm{d} N$. With this approach, it is possible to simulate the experimentally observed decreasing crack growth rate when the crack tip approaches grain boundaries. The crack tip slide displacements as well as the crack growth rate decrease until the critical shear stress for slip transmission through grain boundaries $\left(\tau^{c}\right)$ is exceeded resulting in new shear band nucleation in the neighbouring grain and in a sudden additional plastic deformation along these newly created shear bands that further increases the crack tip slide displacement. Besides to the determination of the crack growth rate, the direction of the crack advance has to be modelled. It is assumed that the crack propagates in the direction of the activated shear bands by vectorially adding the corresponding deformation components along the active slip systems.

If the crack tip reaches the grain boundary, it can further propagate either along this grain boundary or into the next grain by activating new shear bands. This problem is solved by introducing the critical normal stress for crack growth along a grain boundary $\left(\sigma^{\mathfrak{c}}\right)[15]$. If the critical stress for nucleation of shear bands as wells as for crack growth along a grain boundary is exceeded, the condition $\xi=\tau^{\mathrm{c}} / \sigma^{\mathrm{c}} \cdot\left|\sigma^{\mathrm{GB}} / \tau^{\mathrm{MRSS}}\right|$ determines the new crack path with $\sigma^{\mathrm{GB}}$ as the calculated normal stress along the specific grain boundary and $\tau^{\text {MRSS }}$ represents the maximum resolved shear stress in the next grain. Thereby, for $\xi>1$, the crack follows the grain boundary.
To model the crack growth along grain boundaries, a similar approach to eq. (1) is used. The crack growth rate in this case is calculated by the relative crack tip opening displacement $(\triangle C T O D)[15]$

$$
\frac{\mathrm{d} a}{\mathrm{~d} N}=C_{\mathrm{CTOD}} \cdot \triangle C T O D
$$

where the parameter $C_{\mathrm{CTOD}}$ is again a measure for the irreversible deformation processes at the crack tip. The crucial parameter for crack growth along grain boundaries is the crack tip opening displacement. This approach can be justified by Fig. 2e where the crack propagates in the normal direction to the applied exterior load. This results in increasing normal stresses along the grain boundary such that the tangential component of the crack displacement becomes insufficient for describing the crack growth processes.

The plastic deformations at the vicinity of the grain boundary crack is modelled according to the Dugdale model [28]. The plastic zone is projected to yield strips along the active grain boundary [15]. The condition for yielding is met if the von-Mises stress $\left(\sigma^{\mathrm{vM}}\right)$ exceeds the cyclic flow stress $\left(\sigma^{\mathrm{F}}\right)$ of the material.

The crack growth mechanisms described above are assumed to be valid in the presence of hydrogen as can be observed in Fig. 2a-e. The simulation of short cracks based on the presented mechanisms will further reveal the acting hydrogen related mechanisms during short crack propagation.

To solve the model, an elastostatic simulation is carried out and solved numerically with the boundary element method (BEM). In this simulation, the fatigue loading is sequentially applied. During each loading step, the microstructure is iteratively solved and adjusted e. g. in the case of new shear bands nucleated at the crack tip or the iterative determination of the plastic deformation along shear bands or grain boundaries. The boundary element method used for this simulation is presented in the following section.

\section{Numerical method}

Using the boundary element method compared to the finite element method (FEM) has several advantages in the case for solving the short crack propagation. One obvious reason is the effort for discretization which is significantly less compared to the FEM. Especially when taking a closer look at the modelled deformation processes in the microstructure which are restricted to the representative shear bands such that only the solution at these sites are relevant. The elastic solution inside a grain is obtained by only discretizing the grain boundary $\left(I^{b}\right)$. Furthermore, with the used BEM the relative displacements and stresses in the crack elements as well as along shear bands can directly be calculated. In the presented BEM, only the upper flank $\left(\Gamma^{\mathrm{s}^{+}}\right)$of the crack or a shear band is modelled. Thus, to calculate displacements and stresses, the following displacement and stress boundary integral equations have to be solved where greek subscripts take the values 1 or 2 and latin subscripts are in the range from 1 to 3. 


$$
\begin{aligned}
& c_{i j} u_{i}=\int_{\Gamma^{\circ}}\left[u_{i j}^{*} t_{\mathrm{i}}-t_{i j}^{*} u_{i}\right] \mathrm{d} \Gamma^{\mathrm{b}}+\int_{\Gamma^{s+}} t_{i j}^{*} \Delta u_{i} \mathrm{~d} \Gamma^{\mathrm{s}+} \\
& \sigma_{j \gamma}=\int_{\Gamma^{\mathrm{b}}}\left[d_{i j \gamma}^{*} t_{i}-s_{i j \gamma}^{*} u_{i}\right] \mathrm{d} \Gamma^{\mathrm{b}}+\int_{\Gamma^{\mathrm{b}}} s_{i j \gamma}^{*} \Delta u_{i} \mathrm{~d} \Gamma^{\mathrm{s}+}
\end{aligned}
$$

By defining appropriate boundary conditions, eq. (3) is used to calculate the displacements $u_{i}$ and the boundary stresses $t_{i}$ along the discretized grain boundaries as well as the relative displacements $\Delta u_{\mathrm{i}}$ along the crack and shear band elements. In a post-processing stage, the stresses $\sigma_{j y}$ inside the grains are determined. In eq. (3), $u_{i j}^{*}$ and $t_{i j}^{*}$ are the displacement and traction fundamental solutions both with a weak singularity and in eq. (4) $d_{i j \gamma}^{*}$, and $s_{i j \gamma}^{*}$ are the stress and the higher-order stress fundamental solutions. These are given by Wang [29] who derived these fundamental solutions for a general anisotropic elastic solid.

With the general plane stress assumption, the shear deformations and stresses in the slip bands can be calculated in the true direction of the activated slip system.

In the present study, the collocation formulation of the boundary element method is used. In contrast to the Galerkin formulation, the test function is defined by the Dirac function. The evaluation of the integrals in eq. (3) and (4) are obtained analytically by discretizing the grain boundary as well as the crack or shear bands by elements with quadratic shape functions [17].

In the case of modelling a polycrystalline microstructure the individual homogeneous grains have to be coupled at the neighbouring boundaries of each grain. In the BEM, this is achieved by enforcing the continuity conditions along these boundaries [30]. A similar approach is used for modelling cracks along grain boundaries such that relative displacements can directly be calculated [16].

By using the presented BEM, it is finally possible to represent and solve the crack growth propagation in a two-dimensional microstructure consisting of individual grains with different anisotropic elastic properties and the changing short crack growth mechanisms in the presence of hydrogen can be observed.

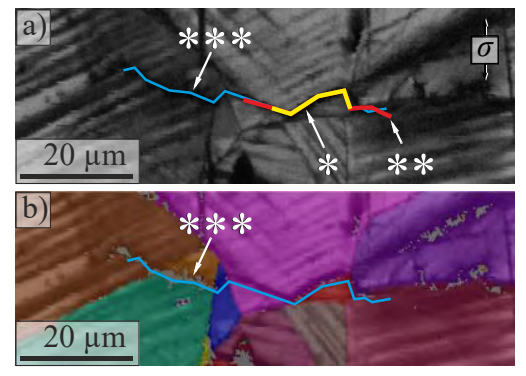

c)

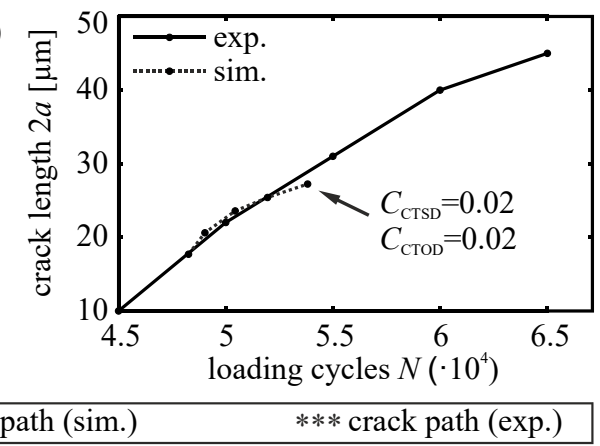

Fig. 3. (a) Crack morphology and (b) EBSD scan of the crack path in an uncharged specimen tested in laboratory air compared with the predicted crack path in the simulation starting from a predefined inital crack and (c) comparison of the calculated crack length $2 a$ related the loading cycles $N$ with experimentally obtained data

\section{Simulation of HCF short crack growth in the presence of hydrogen}

In the following, three different simulations on the short crack propagation are presented and compared with the experimentally observed crack path and crack growth rates, (i) in an uncharged specimen, (ii) in a specimen precharged with hydrogen and (iii) in a specimen tested in $10 \mathrm{MPa}$ hydrogen atmosphere.

To reveal the influence of hydrogen on the short crack propagation, the following modelling strategy is used. At first, the presented crack growth model is validated in the uncharged specimen. Subsequently, simulations in the specimen precharged with hydrogen and in the specimen in $10 \mathrm{MPa}$ hydrogen atmosphere is carried out with the same validated modelling and simulation parameters as in the uncharged case. By adjusting appropriate parameters such that the crack paths as well as the crack growth rates in the presence of hydrogen are correctly predicted, the changing short crack growth mechanisms related to hydrogen will be revealed.

The model parameters chosen to calculate the crack propagation in the uncharged condition are given in Table 2. These parameters can be calculated according to [13-15] with the endurance limit of the material defined as $\sigma^{\mathrm{D}}=250 \mathrm{MPa}$ and the experimentally obtained mean grain size of $D_{\text {mean }}=205 \mu \mathrm{m}$.

Table 2.

Model parameters of microstructural deformation processes

\begin{tabular}{ll}
\hline Parameter & Uncharged condition \\
\hline$\tau^{\mathrm{c}}$ & $108 \mathrm{MPa}$ \\
\hline$\tau^{\mathrm{F}}$ & $80 \mathrm{MPa}$ \\
\hline$\sigma^{\mathrm{c}}$ & $216 \mathrm{MPa}$ \\
\hline$\sigma^{\mathrm{F}}$ & $410 \mathrm{MPa}$ \\
\hline
\end{tabular}

Fig. 3 shows the simulation results obtained in the uncharged condition. In Fig. $3 \mathrm{a}$, it can be seen that the predicted crack path in the simulation correlates well with the experimentally observed crack path. The parameters $C_{\mathrm{CTSD}}$ and $C_{\mathrm{CTOD}}$ describing the irreversible crack growth processes are determined by comparing the predicted crack growth rate with experimentally obtained

\begin{tabular}{|lll}
$*$ crack initiation $(\operatorname{sim})$. & $* *$ crack path $(\operatorname{sim})$. & $* * *$ crack path $($ exp.) \\
\hline
\end{tabular}


results. Similar values for the irreversibility constants are determined by Kübbeler [16] for short crack growth simulations in the metastable austenitic stainless steel X2CrNi18-9 $\left(C_{\mathrm{CTSD}}=0.018\right)$. With these results, the assumptions in the crack growth model can be justified for this material such as the modelling of up to two representative shear bands at each crack tip. Based upon the simulation of the short crack propagation in the uncharged specimen, the changing short crack growth mechanisms in the presence of hydrogen can be discussed.

In Fig. 4, the simulation results of the short crack propagation in the presence of hydrogen are demonstrated. The model parameters given in Table 2 are not adjusted to predict the crack path in the precharged specimen (Fig. 4a). During the early phase of crack growth, the predicted crack path can reasonably be described, but later deviates from the experimentally observed crack path. This can be explained by taking a closer look at the activated shear bands and their deformation during the simulation as further emphasized in Fig. 4a. First of all, it can be seen that the simulation provides a good result of the activated shear bands in comparison with the observed slip markings. The amount of shear deformation along these simulated shear bands is depicted by the size of the straight lines. As stated before, the direction of the crack growth in the simulation is governed by the shear deformation and is obtained by vectorially adding these deformations. The deviating crack path during the later stage of crack growth for example at the left crack tip is a consequence of the underestimation of the slip deformation on the second activated shear band. Nevertheless, during early crack propagation a good agreement can also be obtained
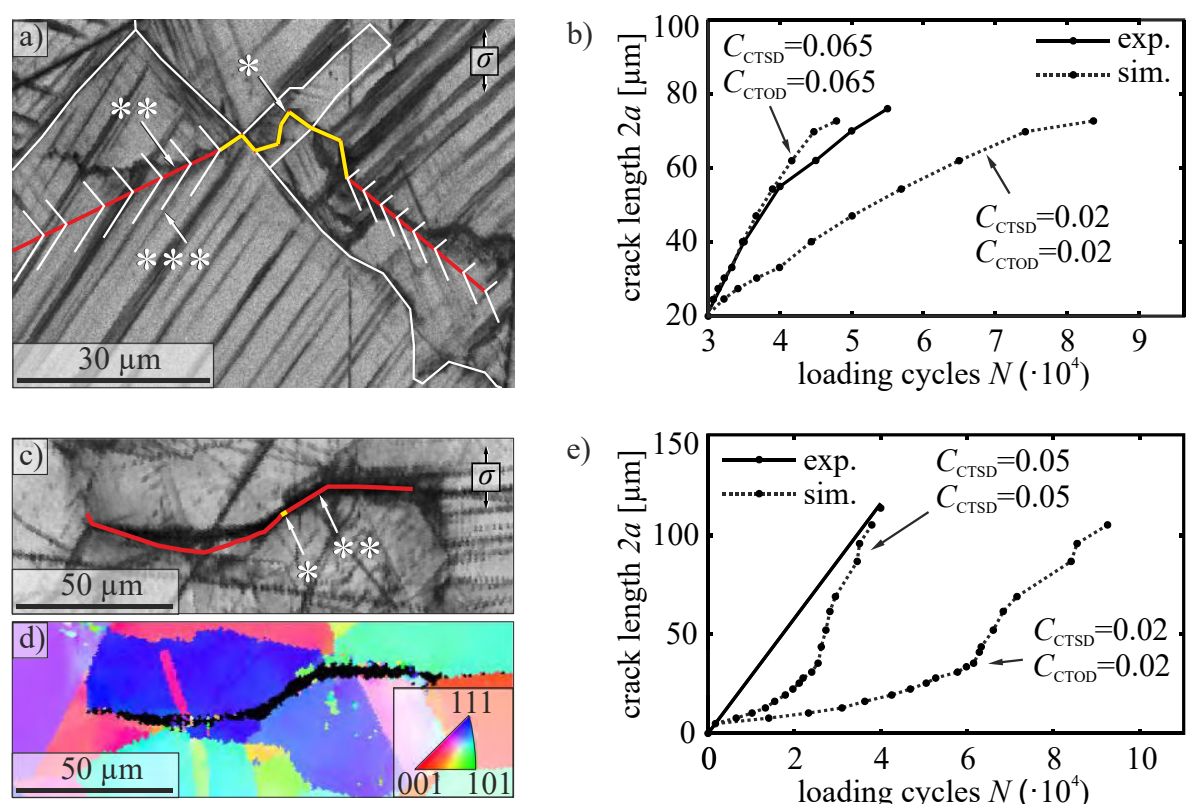

e)

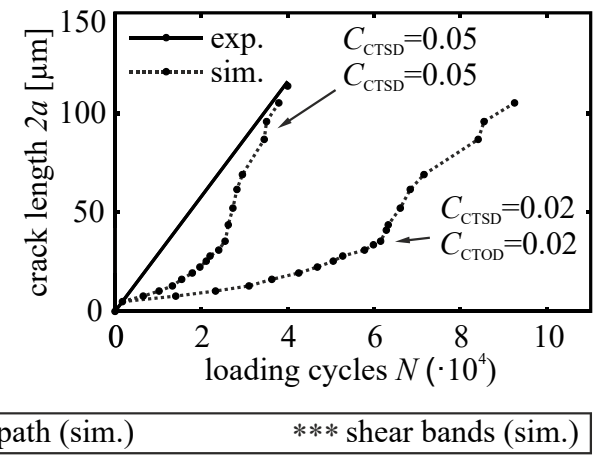

Fig. 4. (a) Crack morphology of the crack path in a precharged specimen $\left(c_{\mathrm{H}}=40 \mathrm{wppm}\right)$ compared with the predicted crack path in the simulation starting from a predefined inital crack, (b) comparison of the calculated crack length $2 a$ related the loading cycles $N$ with experimentally obtained data and (c) crack morphology and (d) EBSD scan of the crack path in a specimen tested in $10 \mathrm{MPa}$ hydrogen atmosphere compared with the predicted crack path in the simulation starting from a predefined inital crack and (e) comparison of the calculated crack length $2 a$ related the loading cycles $N$ with experimentally obtained data 
increased by two mechanisms. At first, the transgranular crack growth is facilitated by the aforementioned hydrogen-enhanced irreversible crack growth processes at the crack tip. And on the other hand, the cracking along the grain boundaries is promoted by hydrogen. This can be explained by the HEDE model. The entry of hydrogen at the grain boundaries is facilitated. As grain boundaries can act as strong barriers, hydrogen atoms tend to reside at these sites leading to an increased local hydrogen concentration. This results in weakening the strength of grain boundaries and promoting hydrogen related cracking, as observed in [1]. Thus, the cause for the increasing irreversibility $C_{\text {CTOD }}$ is the effect of weakened grain boundary strength rather than facilitated irreversible plastic deformation processes at the crack tip as in the case for transgranular crack growth along shear bands.

\section{Conclusions}

In this study, the cyclic short crack propagation of a metastable austenitic stainless steel was investigated in an uncharged specimen, in a specimen precharged with hydrogen and in a specimen in $10 \mathrm{MPa}$ hydrogen atmosphere. The experimental observations were supported by simulations on the short crack growth in these different conditions. From these results, it could be found that the prevailing hydrogen mechanism for short crack growth is dependent on the presence of hydrogen.

1. In precharged specimen, the short crack propagation is predominantly transgranular. The irreversible plastic deformations at the crack tip are locally enhanced by hydrogen according to the hydrogenenhanced localized plasticity (HELP) model. Birnbaum et al. [5] proposed that hydrogen leads to decreased interactions between dislocations and short range stress fields. Based on the simulation results, it could be found that hydrogen enhances the short crack growth mechanisms as stated by Wilkinson et al. [4] where the crack propagates by absorbing dislocations or vacany dipoles at the crack tip and further results in an increased fatigue crack growth rate.

2. In specimen tested in $10 \mathrm{MPa}$ hydrogen atmosphere, the short crack propagation is predominantly intergranular. The simulation supports the prevailing hydrogen-enhanced decohesion (HEDE) model such that hydrogen promotes grain boundary cracking in the investigated metastable austenitic stainless steel. Furthermore, hydrogen also increases the irreversible crack growth process in the case for transgranular crack propagation in this condition.

\section{Acknowledgement}

The authors gratefully acknowledge financial support of this study by Deutsche Forschungsgemeinschaft (DFG) and Forschungsvereinigung Verbrennungskraftmaschinen e.V. (FVV). Reference numbers: CH92/48-1 (DFG) and 1185 (FVV).

\section{References}

1. S. Fukuyama, K. Yokogawa, K. Kudo, M. Araki, Trans. Jpn. Inst. Met., 26, 5, 325-331 (1985)

2. Y. Murakami, T. Kanezaki, Y. Mine, S. Matsuoka, Metall. Mater. Trans. A, 39A, 1327-1339 (2008)

3. H. H. Heitmann, H. Vehoff, P. Neumann, Pergamon Press Ltd. 5, 599-606 (1984)

4. A. J. Wilkinson, S. G. Roberts, Scripta Mater. 35, 1365-1371 (1996)

5. H. K. Birnbaum, P. Sofronis, Mater. Sci. Engng. A176, 191-202 (1994)

6. A. R. Troiano, Trans. ASM 52, 54-80 (1960)

7. R. A. Oriani, P. H. Josephic, Acta Metall. 25, 979-988 (1977)

8. R. A. Oriani, Corrosion 43, 390-397 (1987)

9. M. Nagumo, Mater. Sci. Tech. 20, 940-950 (2004).

10. M. Hatano, M. Fujinami, K. Arai, H. Fujii, M. Nagumo, Acta Mater. 67, 342-353 (2014)

11. S. Lynch, Corrosion Reviews 30 (3-4), 105-123 (2012)

12. S. Matsuoka, N. Tsutsumi, Y. Murakami, Trans. Jpn. Soc. Mech. Eng. A 74 (748), 44-53 (2008)

13. A. Schick, Dissertation, Universität Siegen, 2004

14. B. Künkler, Dissertation, Universität Siegen, 2007

15. P. Köster, Dissertation, Universität Siegen, 2015

16. M. Kübbeler, Dissertation, Universität Siegen, 2017

17. P.-M. Hilgendorff, Dissertation, Universität Siegen, 2017

18. F. B. Pickering, Proc. Conf. Stainl. Steels 84, 2-28 (1984)

19. K. Nohara, Y. Ono, N. Ohashi, J. Iron Steel Inst. Jpn. 63, 212-222 (1977)

20. I. Roth, Dissertation, Universität Siegen, 2011

21. T.-P. Perng, C. J. Altstetter, Acta metall. 34 (9), 1771-1781 (1986)

22. A. Heinz, P. Neumann, Acta Met. Mat. 38, 1993-1940 (1990)

23. I. Roth, M. Kübbeler, U. Krupp, H.-J. Christ, C.-P. Fritzen, Proc. Engn. 2 (1), 941-948 (2010)

24. S. Brück, V. Schippl, C.-P. Fritzen, H.-J. Christ, M. Schwarz, S. Weihe, ASME, 297-306 (2017)

25. I. Aubert, N. Saintier, J. M. Olive, Scripta Mater. 66 (9), 698-701 (2012)

26. D. P. Abraham, C. J. Altstetter, Metall. Mater. Trans. 26A, 2859-2871 (1995)

27. A. Navarro, E. R. de los Rios, Phil. Mag. A 57 (1), 15-36 (1988)

28. D. S. Dugdale, J. Mech. Phys. Solids 8, 100-104 (1960)

29. C.-Y.Wang, Int. J. Solids Struct. 31, 2591-2597 (1994)

30. M. Kübbeler, I. Roth, U. Krupp, C.-P. Fritzen, H.-J. Christ, Eng. Fract. Mech. 78, 462-468 (2011)

31. K. Nygren, A. Nagao, P. Sofronis, I. M. Robertson, ASME, 270-277 (2017)

32. M. L. Martin, P. Sofronis, I. M. Robertson, T. Awane, Y. Murakami, Int. J. Fat. 57, 28-36 (2013) 